Rev. Biol. Trop., 47(1-2): 109-117, 1999

www.ucr.ac.cr www.ots.ac.cr www.ots.duke.edu

\title{
Periodo reproductivo y talla de madurez masiva del camarón de roca Sicyonia penicillata (Decapoda: Sicyoniidae) en Bahía Kino, Sonora, México
}

\author{
J. López-Martínez¹, F. García-Domínguez², E. Alcántara-Razo y E. A. Chávez² \\ 1 Centro de Investigaciones Biológicas del Noroeste, S.C. Unidad Guaymas. Apdo. Postal 349. Guaymas, Sonora, \\ 85454, México. Fax. (622) 122-38, Internet: jlopez@ cibnor.mx \\ 2 Centro Interdisciplinario de Ciencias Marinas, Instituto Politécnico Nacional. Apdo. Postal 592. La Paz, B.C.S., \\ 23000, México.
}

Recibido 10-VII-1998. Corregido 7-XI-1998. Aceptado 9-XI-1998.

\begin{abstract}
The reproductive pattern of the rock shrimp (Sicyonia penicillata) was studied from January through December (1996) by sampling from boats twice a month (commercial shrimp fleet and one research cruise). Reproductive activity begins at $25 \mathrm{~mm}$ of tail length, but massive maturity or critical size is $55 \mathrm{~mm}$ of tail length. Mature organisms were found year round, but recruitment pattern analysis indicates that massive reproduction is from August through January; a second, less intense reproductive peak, occurs in March-April. Results are coincident with the gonadical maturity analysis. A migration pattern of juveniles towards shallow areas of Bahía Kino is suggested.
\end{abstract}

Key words: Shrimp, Sicyoniidae, Sicyonia penicillata, reproduction, growth, recruitment.

En el área de Bahía Kino, Sonora (Fig. 1) aparece como captura incidental en los arrastres de camarones del género Penaeus, una especie de camarón del género Sicyonia, Sicyonia penicillata (Lockington 1879), conocida como camarón de roca, cacahuate o japonés, la cual, por los volúmenes que se obtuvieron en las temporadas 1994-1995, 1995-1996 y 19961997 (650, 3415 y 769 toneladas métricas, respectivamente) ha despertado el interés del sector pesquero de la región, que actualmente ha solicitado permisos para la pesca de esta especie en periodos de veda de los camarones del género Penaeus. Este hecho ha generado la necesidad de contar con información sobre los aspectos básicos de la biología de Sicyonia penicillata.

Pocos estudios se han hecho a nivel mundial de las especies de este género, y de éstos

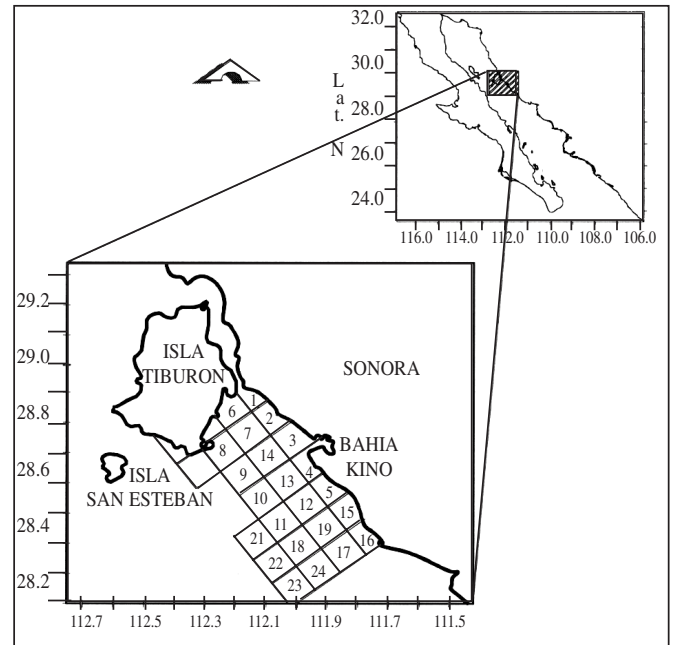

Fig. 1. Estaciones prospectadas durante el crucero de investigación de camarón de roca Sicyonia penicillata efectuado a bordo del B/O BIP II durante el mes de julio de 1996 en Bahía Kino, Sonora, México. 
algunos tratan sobre su descripción a nivel de especie y distribución batimétrica y geográfica (Hendrickx 1984, Freitas 1984, Pérez-Farfante 1985, Sunada y Richards 1992), comportamiento (Bauer 1985), bioquímica y comportamiento reproductivo (Kleve et al. 1980, Anderson et al. 1985, Wikramanayake y Clark 1995, Subramoniam 1995), y análisis del potencial pesquero (Frey 1971, Cobb et al. 1973, Arreguín-Sánchez 1981, Smith 1988).

Unos de los conocimientos biológicos básicos necesarios para administrar una pesquería es la edad de maduración masiva (la edad en que el $50 \%$ de la población está madura) y el periodo reproductivo, cuyo conocimiento determina en alto grado las medidas regulatorias que se adopten para la administración.

Las especies del género Sicyonia presentan sexos separados, con cópula múltiple por un mismo macho y aún la cópula de varios machos en una misma hembra (Sunada y Richards 1992, Bauer 1992). Ambos sexos pueden madurar sexualmente antes de cumplir su primer año de vida, pero la mayor parte de la población madura durante el segundo año a una talla de 25-30 mm de longitud del cefalotórax (LC) (Kennedy et al. 1977, Sunada y Richards 1992). En cuanto a los periodos reproductivos se han registrado especies con hembras maduras sexualmente a lo largo del año y con uno o dos periodos masivos, tales como $S$. ingentis y S. brevirostris (Kennedy et al. 1977, Anderson et al. 1984, Anderson et al. 1985, Clark y Pillai 1991, Sunada y Richards 1992), y especies con reproducción contínua sin un aparente periodo de reproducción masiva como S. parri y S. laevigata (Bauer y Rivera-Vega 1992). En S. ingentis el número promedio de óvulos producidos por hembra por desove llega a ser del orden de 86000 (Sunada y Richards 1992).

En $S$. penicillata es muy poco lo que se conoce de su biología, salvo que es una especie endémica del Golfo de California, con las mayores concentraciones entre los 60 y 103 m de profundidad (Hendrickx 1984). Por lo anterior, el objetivo principal del presente trabajo es determinar la longitud de maduración masi- va o talla crítica, así como los periodos de reproducción del camarón de roca Sicyonia penicillata, con la finalidad de contribuir a sentar las bases para una administración adecuada del recurso, ante la eventual apertura de una pesca dirigida a esta especie.

\section{MATERIALES Y MÉTODOS}

La información que sirvió de base para el presente trabajo provino de dos fuentes diferentes:

- Muestreos quincenales simultáneos a bordo de tres embarcaciones camaroneras de la flota comercial que opera con redes de arrastre de $20 \mathrm{~m}$ de abertura de boca en la relinga superior y luz de malla de $5.71 \mathrm{~cm}$ en cuerpo y alas y $4.45 \mathrm{~cm}$ en el copo, en el área de Bahía Kino, en el Golfo de California (Fig. 1), de enero a abril y de septiembre a diciembre de 1996, realizándose en total 15 muestreos. De mayo a la primera quincena de septiembre no se hicieron muestreos en la flota comercial debido a que es el período de veda de la pesquería de camarón en el Pacífico mexicano. Los muestreos fueron nocturnos, entre las 18:00 y las 05:00 hr, que es el horario en que se efectúa la pesca, a una profundidad aproximada de 14-18 m. Durante la noche se efectuaron al menos dos lances con una duración de 3 a 4 hr y de cada lance se obtuvo una muestra de la captura sin seleccionar de $10 \mathrm{~kg}$ aproximadamente. Los ejemplares de camarón de roca fueron separados y congelados para su análisis en el laboratorio.

- Debido a que desde mayo y hasta principios de septiembre no opera la flota comercial, durante el mes de julio se realizó un crucero de investigación a bordo del B/O BIP II perteneciente al Centro de Investigaciones Biológicas del Noroeste, S.C. El muestreo fue sistemático siguiendo el derrotero que se esquematiza en la figura 1. La prospección se efectúo de 9 a 
$90 \mathrm{~m}$ siguiendo transectos con cuadrantes de 10 X $10 \mathrm{~km}$. Las muestras fueron obtenidas con redes camaroneras tipo mixto, que es el tipo de red comúnmente usada por los barcos camaroneros de la zona para la pesca de camarón de roca, de tal manera que las muestras fueran comparables. El tratamiento de las muestras fue el mismo que el descrito previamente para las muestras de las capturas comerciales.

En el laboratorio los organismos se identificaron y se les determinó el peso total, longitud total $\left(\mathrm{L}_{\mathrm{t}}\right)$, longitud abdominal $\left(\mathrm{L}_{\mathrm{a}}\right)$, sexo y madurez gonadal, ésta última siguiendo la escala morfocromática descrita en el Cuadro I, la cual se basa en el aspecto de los óvulos y la coloración de los ovarios (Barreiro 1986). Para la identificación de los organismos se utilizaron las claves propuestas por Pérez-Farfante (1985) y Hendrickx (1995), cuyos ejemplares de referencia se encuentran depositados en la Colección de Referencia de Invertebrados de la Estación Mazatlán del Instituto de Ciencias del Mar y Limnología de la Universidad Nacional Autónoma de México. El tamaño al que los machos alcanzan la madurez sexual se determinó usando como criterio la fusión de los endopoditos del primer par de pleópodos (Pérez-Farfante 1969). El tamaño en el que la es- pecie alcanza la madurez sexual se obtuvo de la relación entre la fracción acumulada de las hembras maduras en estadios tres, cuatro y cinco (madurez gonadal avanzada, en desove y desovados respectivamente) a una cierta longitud, contra la longitud. Dicha relación describe una curva de tipo logístico. La talla de maduración masiva se describe como aquel punto que intercepta el $50 \%$ de la curva acumulada (Sparre y Venema 1995).

Con los datos de frecuencia de tallas (considerando la longitud abdominal) en periodos mensuales, se estimó la curva de selección a través del método de curva de captura linearizada (Sparre y Venema 1995) con la que se ajustó la muestra de frecuencia de tallas para eliminar el sesgo en las muestras generado por la selectividad del arte. Posteriormente se estimaron los parámetros del modelo de crecimiento de von Bertalanffy, que ha sido demostrado que se ajusta bien al crecimiento en camarones (Ricker 1975, Pauly 1984, Parsons et al. 1986)

$$
\begin{aligned}
& \mathrm{L}_{\mathrm{t}}=\mathrm{L}_{\infty}\left(1-\mathrm{e}^{-\mathrm{K}\left(\mathrm{t}-\mathrm{t}_{\mathrm{O}}\right)}\right) \\
& \text { donde: } \\
& \mathrm{L}_{\mathrm{t}}=\text { longitud a la edad } \mathrm{t} \\
& \mathrm{L}_{\infty}=\text { longitud asintótica } \\
& \mathrm{K}=\text { coeficiente de crecimiento } \\
& \mathrm{t}_{\mathrm{O}}=\text { edad teórica a la que el organismo } \\
& \quad \text { tiene una longitud igual a cero }
\end{aligned}
$$

\section{CUADRO 1}

Escala utilizada para la determinación del estadio de madurez gonadal (Barreiro 1986)

$\begin{array}{llll}\text { Etapa } & \text { Coloración } & \text { Aspecto macroscópico } & \text { Aspecto citológico } \\ \text { I Inmaduro } & \text { Transparente } & \text { Delgada, confinada al abdomen } & \begin{array}{l}\text { Células germinales, } \\ \text { núcleo visible, citoplasma claro }\end{array} \\ \text { II Desarrollo } & \text { Blanco } & \begin{array}{l}\text { Más gruesa, lóbulos } \\ \text { cefalotorácicos incipientes }\end{array} & \text { Ovocitos con núcleo visible } \\ \text { III Madurez avanzada } & \text { Verde claro } & \begin{array}{l}\text { Muy gruesa, lóbulos } \\ \text { cefalotorácicos desarrollados }\end{array} & \begin{array}{l}\text { Ovulos con vitelo, } \\ \text { núcleo oculto }\end{array} \\ \text { IV Madurez completa } & \text { Verde obscuro } & \begin{array}{l}\text { Muy gruesa y granulosa. } \\ \text { Ocupa todo el cefalotórax }\end{array} & \text { Ovulos con vitelo } \\ \text { V Desove } & \text { Transparente } & \text { Flácida y delgada } & \begin{array}{l}\text { Células germinales, } \\ \text { óvulos maduros residuales }\end{array}\end{array}$


Los valores estimados de estos parámetros se obtuvieron mediante los métodos de Shepherd (Shepherd 1987, Pauly y ArreguínSánchez 1995), Electronic LEngth Frecuency ANalysis ELEFAN I (Pauly 1984) y Powell-Wetherall (Powell 1979, Wetherall et al. 1987) utilizando el paquete computacional FiSAT (Gayanilo et al. 1995). El valor del parámetro de condición inicial (to) se obtuvo mediante la ecuación empírica propuesta por Pauly (1980). Una vez obtenidos los parámetros de longitud asintótica $\left(\mathrm{L}_{\infty}\right)$, coeficiente de crecimiento $(\mathrm{K}) \mathrm{y} \mathrm{t}_{\mathrm{o}}$, $\mathrm{y}$ con la finalidad de tener un estimado indirecto basado en las tallas presentes en las capturas del periodo reproductivo, se estimó el patrón de reclutamiento de la especie, utilizando para ello ELEFAN II, el cual incorpora una rutina que con los datos de frecuencia de longitudes presentes en las muestras, y con el sesgo de la selección ya corregido, proyecta hacia atrás en el eje del tiempo (por medio de los parámetros de crecimiento) generando patrones de reclutamiento que pueden ser usados para obtener información objetiva relacionada a la longitud de la temporada de desove, así como a la magnitud relativa de ésta (Pauly 1987).

\section{RESULTADOS}

Se efectuó un total de 15 muestreos en la flota camaronera, de los cuales se obtuvieron 46 muestras. De los 15 muestreos, 11 fueron positivos (aquellos en los que se obtuvo al menos 1 ejemplar de camarón de roca). Si bien la temporada de pesca de camarón en el Pacífico mexicano inició la segunda quincena de septiembre, los muestreos de septiembre, el de la primera quincena de octubre, los dos de noviembre y el segundo de diciembre fueron negativos. Durante el crucero realizado en julio se efectuaron siete lances, de los cuales solamente dos fueron positivos (estaciones 20 y 21), los dos a profundidades mayores de 55 m (Fig. 1).

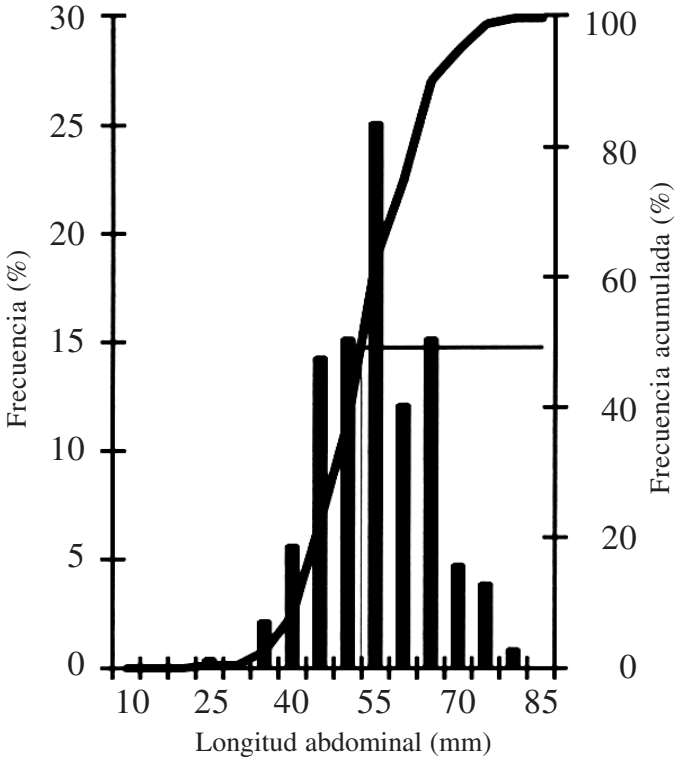

Fig. 2. Distribución de tallas y ojiva de madurez gonádica para determinación de talla de maduración masiva de camarón de roca Sicyonia penicillata en el área de Bahía Kino, Sonora, México.

En total se analizaron 1783 ejemplares. De éstos, 907 fueron hembras y 876 machos. La proporción de sexos hembra : macho fue de 1:0.97. Se presentaron hembras grávidas desde

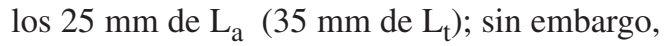
la talla de maduración masiva para la especie (aquella en la que el 50\% de las hembras se encuentran en estadio tres o mayor de tres) se estimó en $55 \mathrm{~mm}$ de $\mathrm{L}_{\mathrm{a}}\left(80 \mathrm{~mm} \mathrm{de} \mathrm{L}_{\mathrm{t}}\right.$ ) (Fig. 2). Entre los machos se encontraron ejemplares con el petasma bien desarrollado desde los 25 $\mathrm{mm}$ de $\mathrm{L}_{\mathrm{a}}$.

Durante todos los meses analizados se observaron hembras que presentaron un cierto desarrollo de ovarios (de dos o mayor), registrándose las mayores proporciones para el mes de octubre (94\%). De enero a la primera quincena de marzo se observó una disminución gradual de la proporción de hembras maduras (Cuadro 2, Fig. 3), la que se volvió a incrementar ligeramente durante la primera quincena de abril y disminuyó al valor mas bajo del año en la segunda quincena del 


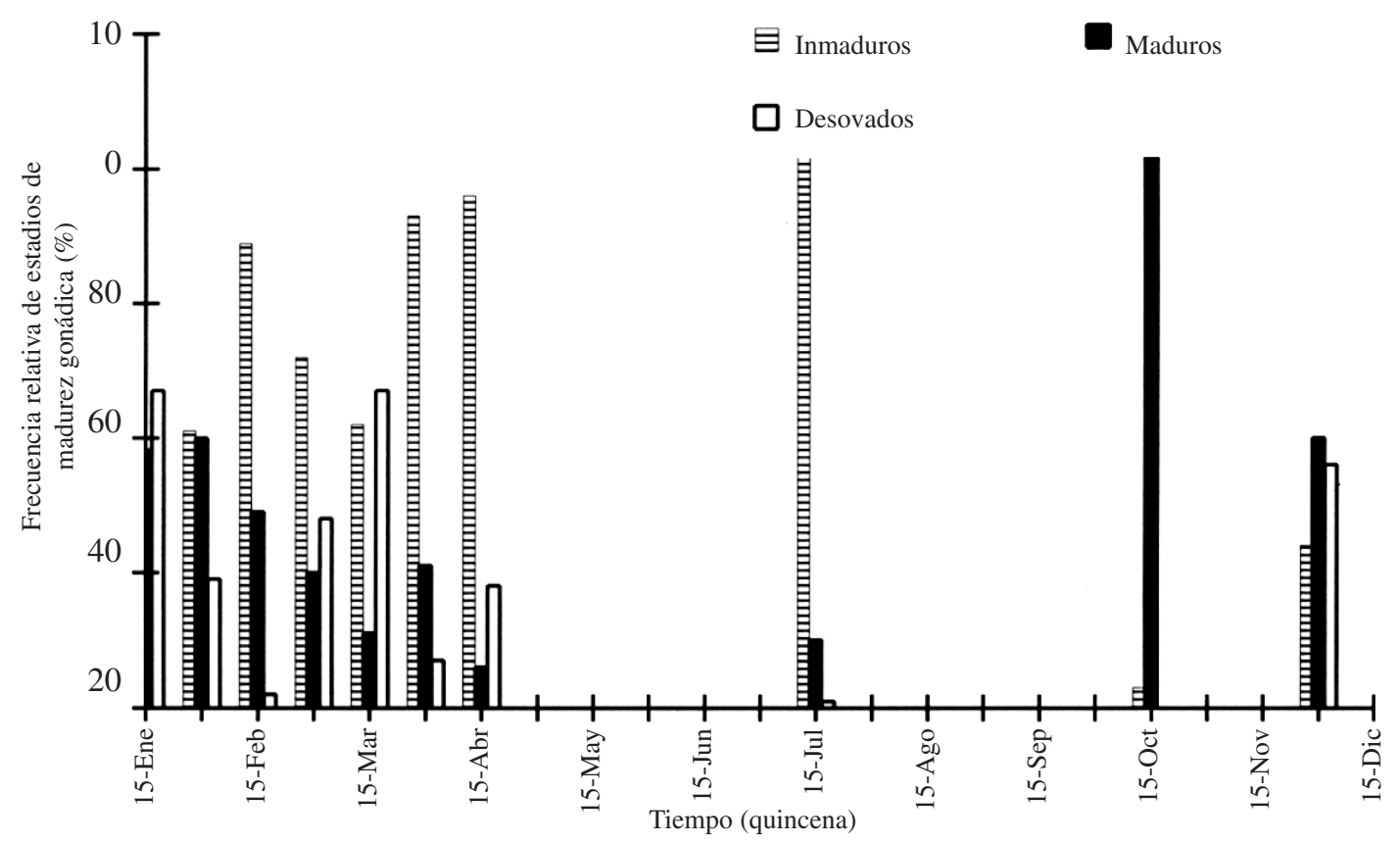

Fig. 3. Frecuencia quincenal de hembras inmaduras (estadio 1), maduras (estadios 2, 3 y 4) y en desove (estadio 5) de camarón de roca Sicyonia penicillata en el área de Bahía Kino, Sonora, México.

CUADRO 2

Frecuencia relativa y número de muestras de organismos de camarón de roca Sicyonia penicillata por fase de madurez gonádica en cada muestreo positivo. Fechas en día/mes

\begin{tabular}{|c|c|c|c|c|c|c|c|c|c|c|}
\hline Fase & 16 & 1 & 16 & 1 & 16 & 1 & 16 & 16 & 16 & 1 \\
\hline & Ene & Feb & Feb & Mar & Mar & Abr & Abr & Jul & Oct & Dic \\
\hline I & 14 & 41 & 69 & 52 & 42 & 73 & 76 & 89 & 3 & 24 \\
\hline II & 8 & 37 & 25.5 & 17 & 9 & 14 & 4 & 8 & 30 & 2 \\
\hline III & 24 & 1 & 3.5 & 0 & 0 & 7 & 2 & 2 & 60 & 29 \\
\hline IV & 6 & 2 & 0 & 3 & 2 & 0 & 0 & 0 & 7 & 9 \\
\hline V & 47 & 19 & 2 & 28 & 47 & 7 & 18 & 1 & 0 & 36 \\
\hline Muestras & 6 & 6 & 6 & 6 & 6 & 6 & 6 & 2 & 2 & 2 \\
\hline
\end{tabular}

mismo mes. Por otra parte los organismos desovados se observaron durante diciembre y de enero a abril, no así durante el mes de julio, en el que el $89 \%$ de los organismos presentes fueron organismos grandes $\left(\mathrm{L}_{\mathrm{a}} 90\right.$ $\mathrm{mm}$ ) e inmaduros (Fig. 3).

El método de Powell-Wetherall subestimó la longitud asintótica $\mathrm{L}_{\infty}(93.33 \mathrm{~mm})$, ya que en los muestreos se encontraron ta- llas mayores a $100 \mathrm{~mm}$. Los otros dos métodos empleados en los análisis mostraron resultados similares tanto para $\mathrm{L}_{\infty}$, como para $\mathrm{K}$ (Cuadro 3). Los valores de $\mathrm{L}_{\infty}=$ $103.9 \mathrm{~mm}$ y $\mathrm{K}=1.24 /$ anual fueron los que se utilizaron para los demás análisis, quedando la ecuación:

$$
\mathrm{L}_{\mathrm{t}}=103.9\left(1-\mathrm{e}^{-1.24 *(\mathrm{t}+0.124)}\right)
$$


CUADRO 3

Parámetros de crecimiento.

$\begin{array}{lcc}\text { Método } & \mathrm{L}_{\infty}(\mathrm{mm}) & \text { K/año } \\ \text { Powell-Wetherall } & 93.33 & 1.47 \\ \text { Shepherd } & 104.0 & 1.20 \\ \text { ELEFAN } & 103.9 & 1.24\end{array}$

Empirical equation of Pauly

Condición inicial $\left(\mathrm{t}_{\mathrm{O}}\right)$, longitud asintótica $\left(\mathrm{L}_{\infty}\right)$ y coeficiente de crecimiento $(\mathrm{K})$ obtenidos mediante los métodos de Sheperd (1987, Pauly y Arreguín-Sánchez 1995), ELEFAN (Pauly 1987)y Powell-Wetherall (Powell 1979, Wetherall et al. 1987).

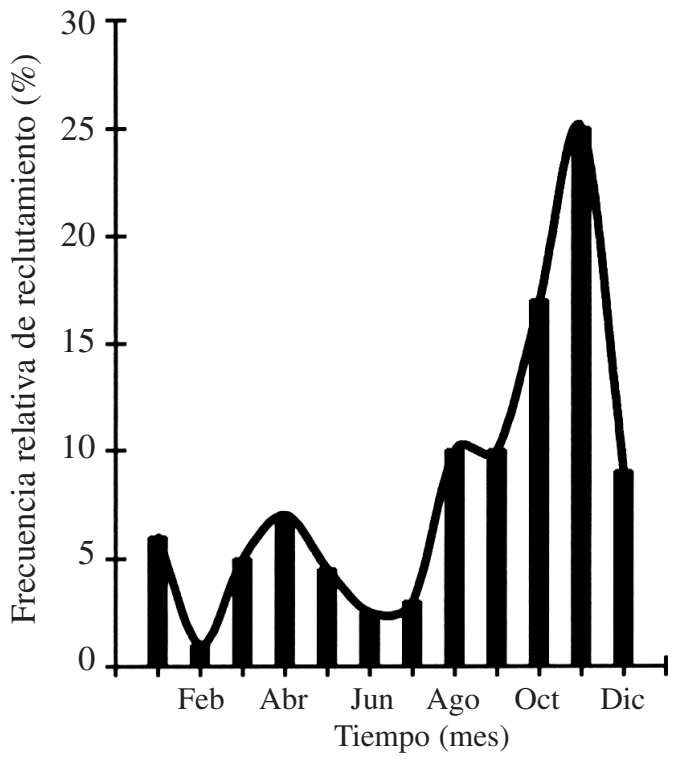

Fig. 4. Frecuencia relativa mensual de reclutamiento del camarón de roca Sicyonia penicillata en Bahía Kino, Sonora, México.

El análisis de patrón de reclutamiento (entendiendo éste como reclutamiento reproductivo) efectuado a través de ELEFAN II (Fig. 4), mostró un reclutamiento contínuo, con un pico máximo que explica el $71 \%$ del reclutamiento anual durante los meses de agosto a diciembre $\mathrm{y}$ un segundo de menor intensidad, que explica el $18 \%$ en marzo-mayo (Fig. 4).

\section{DISCUSION}

La talla de maduración masiva de $S$. penicillata encontrada en este trabajo $(55 \mathrm{~mm}$ de
$\mathrm{L}_{\mathrm{a}} ; 33 \mathrm{~mm}$ de $\mathrm{L}_{\mathrm{c}} ; 80 \mathrm{~mm}$ de $\mathrm{L}_{\mathrm{t}}$ ) coincide con los valores encontrados para otras especies del mismo género (Kennedy et al. 1977, Sunada y Richards 1992). Se observaron diferencias con S. brevirostris (Cobb et al. 1973), especie en la que las hembras maduras más pequeñas miden $46 \mathrm{~mm}$ de longitud total, sin embargo, estos autores señalan que el dato corresponde al de las hembras más pequeñas y no a la longitud de maduración masiva de la población, la cual no se indica.

Los resultados de este trabajo muestran que la reproducción en esta especie es un proceso contínuo y a pesar de la falta de información de madurez gonádica para los meses en que el recurso no es accesible a la flota camaronera, el análisis indirecto efectuado a través del patrón de reclutamiento que de acuerdo con Pauly (1987) puede ser usado para obtener información objetiva relacionada a la longitud de la temporada de desove, así como a la magnitud relativa de ésta, mostró un período de máxima reproducción de agosto a enero y un segundo pico de desove de menor intensidad durante marzo-abril (Fig. 4), que coinciden con lo observado a través del análisis de madurez gonádica (Fig. 3). Este comportamiento es similar al encontrado para S. brevirostris, que presenta un periodo de desove masivo durante los meses de octubre a febrero variando el mes de inicio y de término, aparentemente en respuesta a la duración del fotoperiodo (Cobb et al. 1973, Kennedy et al. 1977) y para S. ingentis, la cual se reproduce de junio a octubre 
(Anderson et al. 1985, Clark y Pillai 1991). La diferencia entre las tres especies es únicamente el inicio del periodo reproductivo. Por otra parte, los resultados aquí encontrados difieren de lo descrito para $S$. parri y $S$. laevigata en Puerto Rico, donde se observan hembras grávidas y organismos juveniles durante todo el año (Bauer y Rivera-Vega 1992).

Si bien los resultados del análisis de madurez gonádica y el de patrón de reclutamiento son coincidentes, las fechas exactas de inicio de los periodos de máxima intensidad están ligeramente desfasadas, lo cual puede ser debido a que los retrocálculos que se efectúan son dependientes, como ya se mencionó previamente, tanto de la estructura de tallas presentes en las capturas, como de la ecuación de crecimiento, la cual incorpora el valor estimado de $t_{0}$, difícil de estimar y que determina en gran medida la asignación de fechas probables de nacimiento de los organismos (Sparre y Venema 1995).

La diferencia entre las profundidades a las que se encontraron los organismos durante los meses de enero a abril y en julio (15-18 $\mathrm{m}$ y mayores de $55 \mathrm{~m}$ respectivamente), además de la casi ausencia durante los meses de septiembre a principios de enero de organismos de $S$. penicillata en las capturas de la flota camaronera que opera en el área, hace suponer que existe un proceso migratorio del recurso hacia áreas no accesibles a la flota, lo que concuerda con lo previamente planteado por ArreguínSánchez (1981), quien ha sugerido que la zona costera de Contoy, Quintana Roo, México, es un área de crianza y reclutamiento de los juveniles de $S$. brevirostris a la pesquería.

Estos resultados no son concluyentes, requiriéndose un muestreo intenso en áreas más profundas de las que opera la flota. Igualmente queda por confirmar si este proceso migratorio realmente ocurre, así como el agente inductor y los periodos de inicio y término de la migración.

\section{AGRADECIMIENTOS}

César A. Salinas Zavala, Rufino Morales Azpeitia y Félix Osuna Villa participaron en los muestreos de campo y en el laboratorio. F. García-Domínguez y E. A. Chávez agradecen el apoyo de la Comisión de Operación y Fomento de Actividades Académicas del Instituto Politécnico Nacional como becarios de exclusividad. Se agradecen los comentarios de dos revisores anónimos.

\section{RESUMEN}

Se estudia el periodo reproductivo del camarón de roca Sicyonia penicillata en el área de Bahía Kino, Sonora, de enero a diciembre de 1996 mediante muestreos quincenales efectuados a bordo de embarcaciones de la flota camaronera comercial y un crucero de investigación durante julio. La actividad reproductiva de la especie inicia a partir de los $25 \mathrm{~mm}$ de longitud abdominal, pero la talla de madurez sexual masiva de la especie se ubicó en $55 \mathrm{~mm}$ de longitud abdominal. Se encontraron organismos maduros todo el año, pero el patrón de reclutamiento indica que existe un periodo reproductivo masivo de agosto a enero; un segundo periodo, menos intenso ocurre en marzo y abril. Estos resultados son coincidentes para los meses en los que se cuenta con datos de madurez gonádica. Se postula la idea de un posible patrón de migración de juveniles hacia áreas someras de Bahía Kino.

\section{REFERENCIAS}

Anderson, S.L., W.H. Clark \& E.S. Chang. 1984. Timing of postvitellogenic ovarian changes in the ridgeback praw, Sicyonia ingentis (Penaeidae) determined by ovarian biopsy. Aquaculture 42:257-271.

Anderson, S.L., W.H. Clark \& E.S. Chang. 1985. Multiple spawning and molt synchrony in free spawning shrimp (Sicyonia ingentis: Sicyoniidae). Biol. Bull. 168:337-394.

Anónimo. 1975. Boletín informativo del Centro Regional de Investigaciones Pesqueras de Guaymas. Instituto Nacional de la Pesca, México. 3.

Arreguín-Sánchez, F. 1981. Diagnosis de la pesquería de camarón de roca (Sicyonia brevirostris Stimpson, 1871) de Contoy, Q. Roo, México. Ciencia Pesquera 1:21-41.

Barreiro, G.M.A. 1986. Estudio sobre la madurez y desove de Penaeus californiensis y Penaeus vannamei (Crustacea Decapoda, penaeidae) en la costa sur de Sinaloa. Memorias del Ier intercambio académico sobre investigaciones en el Mar de Cortés. Abril de 1986. Hermosillo, Sonora México:1-29. 
Bauer, R.T. 1985. Penaeid shrimp fauna from tropical seagrass meadows: species composition, diurnal, and seasonal variation in abundance. Proc. Bio. Soc. Wash. 98:177-90.

Bauer, R.T. 1992. Repetitive copulation and variable success of insemination in the marine shrimp Sicyonia dorsalis (Decapoda: Penaeoidea). J. Crust. Biol. 12:153-160.

Bauer, R.T. \& Rivera-Vega, L.W. 1992. Pattern of reproduction and recruitment in two sicyoniid shrimp species (Decapoda: Penaeoidea) from a tropical seagrass habitat. J. Exp. Mar. Biol. Ecol. 161:223-240.

Clark, W.H. Jr. \& M.C. Pillai. 1991. Egg production, release and activation in marine shrimp, Sicyonia ingentis. Crust. Iss. 7:35-50.

Cobb, S.P., C.R. Furch \& D.K. Camp. 1973. The rock shrimp, Sicyonia brevirostris Stimpson, 1871 (Decapoda, Penaeidae). Mem. Hour. Cruis. Mar. Res. Lab. Fla. 3:1-38.

Freitas, A.J. 1984. The penaeoidea of southeast Africa. VThe family Sicyoniidae. Inv. Rep. South African Assoc. Mar. Biol. Res. 60:1-12.

Frey, H.W. ed. 1971. California living marine resources and their utilization, state of California. the Resources agency, California Department of Fish and Game. 148 p.

Gayanilo, F.C. Jr., P. Sparre \& D. Pauly. 1995. The FAOICLARM Stock Assessment Tools (FiSAT). FAO, Roma. $126 \mathrm{p}$.

Hendrickx, M.E. 1984. The species of Sicyonia H. Milne Edwards (Crustacea: Penaeoidea) of the Gulf of California, México, with a key for their identification and a note on their zoogeography. Biol. Trop. 32: 279-298.

Hendrickx, M.E. 1995. Camarones, p. 512-527. In Fischer, F. Krupp, W. Scheider, C. Sommer, K.E. Carpenter \& V.H. Niem (eds.). Guía FAO para la identificación de especies para los fines de la pesca. Pacífico Centro-Oriental. Vol. 1. Plantas e invertebrados. FAO, Roma.

Kennedy, F.S., J.J. Crane, R.A. Schlieder \& D.G. Barber. 1977. Studies of the rock shrimp Sicyonia brevirostris a new fishery resource on Florida's Atlantic Shelf. Fla. Mar. Res. Pub. 27. 69 p.

Kleve, M.G., A.I. Yudin, W.H. Clark Jr. 1980. Fines structure of the unistellate sperm of the shrimp, Sicyonia ingentis (Natantia). Tissue Cell 12:29-45.
Parsons D.G., G.R. Lilly \& G.J. Chaput. 1986. Age and Growth of northern shrimp Pandalus borealis off northeastern Newfounland and southern Labrador. Trans. Amer. Fish. Soc. 115:872-881.

Pauly, D. 1980. On the interrelationship between natural mortality, growth parameters and mean environmental temperature in 175 fish stocks. J. Conseil. 39:175-192.

Pauly, D., J. Ingles and R. Neal 1984. Application to shrimp stocks of objective methods for the estimation of growth, mortality and recruitment-related parameters from length-frequency data (ELEFAN I and II). In Gulland, J.A. and B. Rothschild (eds.). Penaeid shrimps their biology and management. Fishing News, Oxford, UK.

Pauly, D. 1987. A review of the ELEFAN system for the analysis of length-frecuency data in fish and aquatic invertebrates. ICLARM Conf. Proc. 13:7-34.

Pauly, D. \& F. Arreguín-Sánchez. 1995. Improving Sheperd's length composition analysis (SLCA) Method for Growth parameter estimations. NAGA ICLARM. 18:31-33.

Pérez-Farfante, I. 1985. The rock shrimp genus Sicyonia (Crustacea: Decapoda: Penaeoidea) in the eastern Pacific. Fish. Bull. 1985. 83:1-79.

Powell, D.G. 1979. Estimation of mortality and growth parameters from the length frequency of a catch. Rapp. P. V. Reun. CIEM. 175:167-169.

Ricker, W.E. 1975. Computation and interpretation of biological statistics of fish populations. Fish. Res. Bd. Can. Bull. 19I:382 p.

Smith K. 1988. Grado de conocimiento del recurso camarón del Golfo de México, una perspectiva en la ocasión del 25 aniversario del Instituto Nacional de la Pesca. p. 399-420. In Los recursos pesqueros del país. Secretaria de Pesca, México, D.F.

Sparre P. and S.C. Venema. 1995. Introducción a la evaluación de recursos pesqueros tropicales. Documento Técnico de Pesca 306/I. FAO 177-185 p.

Subramoniam, T. 1995. Ligth and electron microscopic studies on the seminal secretions and the vas deferens of the penaeoidean shrimp, Sicyonia ingentis. J. Biosci. 20:691-706.

Sunada, S. J. \& B.J. Richards. 1992. Ridgeback prawn. p. 11-12. In S. Leet W., C.M. Dewees y C.W. Haugen (eds.). California's living marine resources and their utilization. Sea Grant Extension, Davis, U.S. 
Shepherd, J.G. 1987. A weakly parametric method for estimating growth parameters from length composition data. p. 113-119. In D. Pauly y G.R. Morgan (eds.). Length-based methods in fisheries research. ICLARM Conf. Proc.

Wetherall, J.A., J.J. Polovina \& S. Ralston. 1987. Estima- ting growth and mortality in steady-state fish stocks from length-frecuency data. ICLARM Conf. Proc. 13:53-74.

Wikramanayake, A.H. y W.H. Clark Jr. 1995. Two extracellular matrices from oocytes of the marine shrimp Sicyonia ingentis that independently mediate only primary or secondary sperm binding. Dev. Growth 